\title{
La Relación entre la Salud Oral y el Consumo de Tabaco. Un Estudio de los Habitantes de la Rioja Alta
}

\author{
The Relationship Between Oral Health and Tobacco Consumption. \\ A Study of the Inhabitants of La Rioja Alta
}

José Torres-Remírez¹ \& Marta Ibáñez²

TORRES-REMíREZ, J. \& IBÁÑEZ, M. La relación entre la salud oral y el consumo de tabaco. Un estudio de los habitantes de la Rioja Alta. Int. J. Odontostomat.,14(1):95-100, 2020.

RESUMEN: Estudiar los efectos del consumo de tabaco (producto adictivo) en la salud de los pacientes sigue siendo una prioridad para la sociedad. Tras la lectura de "La Historia Medicinal de las cosas que se traen de nuestras Indias Occidentales" de Nicolás Monardes se realizó una lista de todos los beneficios que se le atribuían al tabaco. Uno de los pocos que no han sido refutados es el del consumo de tabaco para el dolor de muelas. Por ello el objetivo del estudio es relacionar el consumo de tabaco con la salud bucodental (caries) para replicar la afirmación de Monardes. Estudio piloto con pacientes que acuden al dentista en el área de La Rioja Alta (España). Se pidió permiso a los individuos que acudían para tratar sus datos personales en el estudio. Se utilizó estadística descriptiva. Ochenta y tres pacientes cedieron sus datos para la realización del estudio, siendo la mayoría mujeres (59.13\%). La mayoría no son consumidores de tabaco, los consumidores son en su totalidad fumadores diarios. El número medio de caries de la población es de 2.74, siendo el de los fumadores 3.90 y el de los no fumadores 2.10. El número tan bajo de la muestra no permite realizar un estudio de significatividad. Pero, un primer resultado evidencia que los fumadores tienen una peor salud bucodental, lo que los lleva a tener más caries. Esta diferencia no es tan acuciada si se realiza el estudio entre otras variables (sexo, localidad o edad).

PALABRAS CLAVE: tabaco, caries, España.

\section{INTRODUCCIÓN}

El tabaco es un producto adictivo. De dichos productos es el considerado más perjudicial para la salud (Villalbí, 2002). Ni el alcohol, ni los productos con grasas saturadas generan tantos problemas sanitarios y tan diversos como el tabaco. A su vez, el tabaco se relaciona con otros consumos adictivos como el consumo de cánnabis o el incremento del consumo de alcohol, sobre todo en población joven (Míguez Varela \& Becoña, 2015). El tabaco en España representa una media de cincuenta mil muertes al año, es decir, el $13 \%$ del total de las muertes anuales. El consumo está tan extendido que un cuarto de la población nacional fuma diariamente y un $34,7 \%$ de los jóvenes entre 14 a 18 años dice haber fumado alguna vez y sólo un $48,8 \%$ de la población no ha fumado nunca (AECC, 2018).

A pesar de todo el conocimiento que se tiene sobre las consecuencias negativas que provoca el tabaco en la salud de los individuos, aún hay mantras o ideas preconcebidas que son populares sobre los efectos positivos del tabaco. Entre ellos destaca los relacionados con los que minimizan sus consecuencias (Barrado \& Torres-Remírez, 2015). Tras el cáncer de pulmón, pocos conocen la incidencia del tabaco en otros tipos de cáncer como el cáncer de la cavidad nasal y paranasal, nasofaringe, orofaringe e hipofaringe, esófago, vejiga, útero, ovarios, colon o riñón. $Y$ son muchos menos los que conocen su relación con enfermedades orales (Barrado \& TorresRemírez).

Don Nicolás Monardes (1507-1588) fue el primer europeo en escribir sobre las virtudes del tabaco (Torres-Remírez \& Remírez, 2018). Muchas de esas virtudes han quedado como reminiscencia en el conocimiento popular. Una de las virtudes que Nicolás Monardes atribuye al tabaco es su poder analgésico en el dolor de muelas:

\footnotetext{
${ }^{1}$ Universidad de Zaragoza, España.

${ }^{2}$ Clínica Haro Dental, Haro, España.
} 
«En dolor de muelas, quando el dolor es de causa fría o de reumas frías, puesta una pelotilla hecha de la hoja del Tabaco, lavando primero la muela con un pañito mojado en el çumo, quita el dolor y prohíbe no vaya la putrefacion adelante. En causa caliente no aprovecha; y este remedio es ya tan común, que todos sanan» (Monardes, 1992).

Este párrafo demuestra cómo una creencia popular estaba respaldada por argumentos médicos y que ha sido usada por los interesados. $\mathrm{O}$, como unos argumentos médicos se han pervertido hasta convertirse en el mito de que el tabaco es bueno para la salud oral. La labor de los investigadores debe ser desmentir este mito de la virtud del tabaco respecto al dolor de muelas.

El trabajo estará estructurado de la siguiente manera. La siguiente sección se centrará en un estudio de la literatura en el que se ve una relación clara entre el consumo de tabaco y enfermedades orales que provocan un posterior dolor de muelas. A continuación, se presentarán los materiales en los que se basa el estudio piloto relacionando el tabaco con las caries. La caries es una enfermedad oral que no genera un dolor directo, sino que la evolución de la enfermedad acabaría generando dolor permanente e incluso la perdida del diente. En la cuarta sección se verán los resultados del estudio. Las conclusiones del estudio se enumerarán en la última sección.

\section{Revisión de la Literatura}

La literatura sobre los efectos del tabaco se ha centrado en su mayoría en la incidencia sobre el cáncer y posteriormente sobre cómo agrava ciertas enfermedades o la posibilidad de tenerlas. También, las investigaciones se centran en su impacto económico, tanto positivo como negativo. Pero, al final, se ha centrado en asuntos mayoritarios y populares. Otra clase de investigaciones son marginales (Torres-Remírez et al., 2018). Sin embargo, hay ejemplos en la literatura lo suficientemente relevantes como para relacionar el consumo de tabaco con la aparición de enfermedades que causan dolor en la boca.

La Universidad de Ciencias Médicas de Camagüey realizó una revisión de la literatura de todos los artículos publicados en Latinoamérica sobre la evidencia existente entre el consumo de tabaco y la salud oral (Cuba Díaz et al., 2010). Hasta el momento, no se conoce otra revisión más en profundidad de este tema para la región latinoamericana y una de las más completas a nivel mundial. Gracias a esta revisión se puede comprobar la relación del tabaco con enfermedades periodontales más severas, con la halitosis marcada y con el retraso en la cicatrización de las heridas con el consiguiente aumento de las posibilidades de infección. A su vez, también se asocia, aunque en menor medida, con la estomatitis nicotínica palatal, la leucoplasia, la infección gingival, la melanosis, el labio leporino y el paladar fisurado.

A su vez, la revisión de Bertazzo-Silveira et al. (2016) recoge siete estudios con un total de 10.299 pacientes para hacer un metaanálisis y relacionar el bruxismo nocturno con el consumo de productos adictivos. Mientras se encuentra una relación positiva entre el bruxismo nocturno y el consumo de café, alcohol y tabaco, no pasa la mismo al intentar relacionarlo con el consumo de drogas. El estudio no puede desligar el consumo de tabaco y alcohol o tabaco y café, debido al diseño de los estudios que utiliza.

Por último, el flujo salival no estimulado de los consumidores a largo plazo de tabaco entre veinticinco a sesenta años se ve disminuido en un $40 \%$ respecto a la media de flujo salival no estimulado de los pacientes (Gayoso Aguirre, 2017). El flujo salival es una de las variables relevantes a la hora de tratar un paciente, de las posibilidades de sufrir una enfermedad o las probabilidades de curación.

Estos ejemplos escogidos abarcan a su vez una revisión de estudios y de artículos que les impone una representatividad mayor que la de un solo estudio. A su vez, la región en la que están acotados los tres estudios es Latinoamérica, lo que conlleva que la población muestral tenga unos hábitos de higiene, de consumo y de salud propios de la región, que se pueden diferenciar a los de regiones como Asia, Europa o Estados Unidos. Sin embargo, aunque debamos acotar los resultados a la región latinoamericana, queda evidenciado que el consumo de tabaco incide negativamente en la salud oral.

\section{MATERIAL Y MÉTODO}

Muestra. Durante el segundo semestre del 2018 se pidió permiso a los pacientes de la Clínica Dental "Haro Dental" en la localidad de Haro (La Rioja, España) para tratar sus datos y realizar un estudio académico. De todos los pacientes sólo ochenta y tres dieron su autorización para que se trataran sus datos de 
manera anónima para el estudio. La petición se realizó sin informarles sobre el objetivo del estudio ya que se podría desincentivar la participación de los pacientes fumadores o de aquellos cuyo número de caries pudiera ser muy elevado. Estos pacientes están distribuidos geográficamente por toda la región de La Rioja Alta e incluso ciudades colindantes como Miranda de Ebro (Castilla y León) o Vitoria (País Vasco).

Instrumentos. La autorización de los pacientes era la única necesidad que se tenía para poder realizar el estudio. La primera vez que los pacientes acudieron a la clínica se le tomaron los datos necesarios para realizar el estudio. La información de la que se dispone es: la fecha de nacimiento (Edad), el sexo, el domicilio habitual y el consumo de tabaco. Desgraciadamente el consumo de tabaco no está diferenciado por clases (tabaco de liar, de pipa, cigarros, cigarrillos, puros o tabaco de mascar). Esta característica podría haber proporcionado más información, ya que la literatura ha dejado claro las distintas consecuencias para la salud que tienen las clases de tabaco (Torres-Remírez et al.).

Análisis de Datos. Se realizaron análisis estadísticos descriptivos utilizando el programa $R$ versión 3.6.0. y comprobando los datos con el software Gretl para MS Windows.

\section{RESULTADOS}

La muestra que se tiene para realizar el estudio es relativamente homogénea como se puede obser-

Tabla I. Datos Estadísticos de la muestra.

\begin{tabular}{lll}
\hline Sexo & & \\
\hline Hombres & 32 & $38,55 \%$ \\
Mujeres & 51 & $61,44 \%$ \\
Hábitos & & \\
Fumadores & 31 & $37,34 \%$ \\
No Fumadores & 52 & $62,65 \%$ \\
Edad & & \\
Mediana & 37 & \\
Media & 41 & \\
Localidad & & \\
Ciudad & 50 & $60,24 \%$ \\
Pueblo & 33 & $39,76 \%$ \\
\hline
\end{tabular}

var en la Tabla I gracias las características sociodemográficas de los pacientes que acudieron a la consulta.

El objetivo del estudio es ver si los pacientes que acudieron a la consulta y permitieron el uso de sus datos, si se les diferencia por el consumo de tabaco, siguen teniendo una distribución, más o menos homogénea de las caries. En la Tabla II se puede observar el número total de caries que tiene cada subgrupo y las caries medias. A pesar de que es imposible no tener un número entero de caries, se ha decidido desagregar los datos hasta el segundo decimal para poder comprobar mejor la incidencia del tabaco.

Tabla II Salud oral de los pacientes diferenciados por su consumo de tabaco.

\begin{tabular}{lccr} 
& Fumadores & No Fumadores & Total \\
Caries Totales & 121 & 109 & 230 \\
Caries Media & 3,90 & 2,10 & 2,77 \\
\hline
\end{tabular}

Los fumadores son un colectivo menor pero que tienen, tanto en valor absoluto como medio, más caries que los no fumadores. Sin embargo, debemos estudiar el resto de los subgrupos de la muestra para corroborar estos resultados. En las Tablas III, IV y V se puede observar tanto en valores absolutos como medios el número de caries que tienen los pacientes según su sexo, localidad y su edad.

Tabla III. Salud oral de los pacientes según su sexo.

\begin{tabular}{lccc}
\hline & Hombre & Mujer & Total \\
\hline Caries Totales & 94 & 136 & 230 \\
Caries Media & 2,94 & 2,62 & 2,77 \\
\hline
\end{tabular}

Tabla IV. Salud oral de los pacientes según su lugar de residencia.

\begin{tabular}{lccc}
\hline & Ciudad & Pueblo & Total \\
\hline Caries Totales & 141 & 89 & 230 \\
Caries Media & 2,76 & 2,78 & 2,77 \\
\hline
\end{tabular}

Tabla V. Salud oral de los pacientes según la edad.

\begin{tabular}{lcccc}
\hline & $\begin{array}{c}\text { Menores } \\
40 \text { años }\end{array}$ & $\begin{array}{c}40-60 \\
\text { años }\end{array}$ & $\begin{array}{c}\text { Mayores } \\
60 \text { años }\end{array}$ & Total \\
\hline Caries Totales & 137 & 67 & 26 & 230 \\
Caries Media & 3,19 & 2,16 & 2,89 & 2,77 \\
\hline
\end{tabular}


La variable sexo no ha mostrado una desviación de la media de la submuestra a la media de la población muy grande. Lo que está acorde con la literatura de la salud oral y el consumo de tabaco (Bertazzo et al.; Gayoso Aguirre; Torres-Remírez et al.). Respecto a la variable edad muestra una mayor dispersión, sin embargo, sigue siendo menor que la mostrada entre fumadores y no fumadores. En la variable en la que casi no hay diferencia es entre los habitantes de municipios con menos de 10.000 habitantes (pueblos) y los de más de 10.000 (ciudades).

Para poder comprender mejor la relación entre caries y el consumo de tabaco, hace falta realizar un estudio en mayor profundidad. Por ello se desagregarán los datos por el número de caries que se hayan tenido en la Tabla VI.

\section{DISCUSIÓN}

Con los datos que se han obtenido se debería haber podido realizar un estudio de significatividad. Debido a que la muestra (83) era muy recudida, y los subgrupos realizados estaban en su mayoría por debajo del límite de 51 individuos se ha decidido no realizar el estudio de significatividad, ya que la muestra tan reducida no sería idónea para los instrumentos estadísticos. Para la significatividad nos basamos en la idea de que muestras pequeñas (menos de 51 individuos), no son los suficientemente grandes para que los instrumentos sean fiables (TorresRemírez et al.

El principal resultado que se observa es que los fumadores tienen muy pocos individuos con ninguna caries y todos los pacientes con más de diez caries son fumadores. Estos dos aspectos han hecho que el número medio de caries de los fumadores seatan elevado.

A su vez, el resto de las variables no muestran una dispersión tan grande respecto al número de caries medias de la población. Esto demuestra que las diferencias en los hábitos de salud entre hombres y mujeres y entre ciudades y pueblos no distan mucho entre sí. Aunque, se debe estudiar a los fumadores según el resto de las variables para poder tener un mayor conocimiento del escenario tratado. La Tabla VII muestra los resultados:

Tabla VI. Salud oral detallada según el consumo de tabaco.

\begin{tabular}{lccccc}
\hline & Población & Sin Caries & $1-3$ Caries & $4-9$ caries & $>9$ caries \\
\hline Fumadores & $31(37,35 \%)$ & $9(10,84 \%)$ & $11(13,25 \%)$ & $6(7,22 \%)$ & $5(6,02 \%)$ \\
No Fumadores & $52(62,65 \%)$ & $17(20,48 \%)$ & $22(26,50 \%)$ & $13(15,66 \%)$ & $0(0 \%)$ \\
Total & $83(100 \%)$ & $26(31,32 \%)$ & $33(40,75 \%)$ & $19(22,88 \%)$ & $5(6,02 \%)$ \\
\hline
\end{tabular}

Tabla VII. Distribución de los fumadores.

\begin{tabular}{lcccccccc}
\hline & \multicolumn{2}{c}{ Localidad } & \multicolumn{2}{c}{ Sexo } & \multicolumn{2}{c}{ Edad } \\
& Pueblo & Ciudad & Hombre & Mujer & Menor 40 & $40-60$ años & Mayor 60 \\
\hline Fumadores (31) & 16 & 15 & 15 & 16 & 22 & 7 & 2 \\
\hline
\end{tabular}

Tabla VIII Salud oral diferenciando por edad y consumo de tabaco.

\begin{tabular}{|c|c|c|c|c|}
\hline & Menores 40 & $40-60$ años & mayores 60 & Total \\
\hline Caries Totales & 137 & 67 & 26 & 230 \\
\hline Caries Media & 3,19 & 2,16 & 2,89 & 2,77 \\
\hline \multicolumn{5}{|l|}{ Fumadores } \\
\hline Caries Totales & 90 & 29 & 2 & 121 \\
\hline Caries Media & 4,09 & 4,14 & 1 & 3,90 \\
\hline \multicolumn{5}{|l|}{ No Fumadores } \\
\hline Caries Totales & 47 & 38 & 24 & 109 \\
\hline Caries Media & 2,24 & 1,58 & 3,43 & 2,10 \\
\hline
\end{tabular}


El estudio en subgrupos nos ayuda a corroborar como causante de las caries el consumo de tabaco. Tanto por la ubicación de su vivienda habitual, como por el sexo, no se puede deducir nada ya que son distribuciones de la mitad de la población fumadora. Respecto a la distribución por edad, es el colectivo de menos de 40 años los que más caries tienen, y en la distribución de los fumadores, es el colectivo que más fuma. Por lo que hay que tener en cuenta esta cuestión para ver si existe una diferencia en este colectivo entre fumadores y no.

En la Tabla VIII se puede observar cómo la salud oral de los no fumadores es mejor hasta los sesenta años. A partir de los sesenta años los fumadores de la muestra tienen un número menor de caries. Este resultado sí apoya que el deterioro de la salud oral es más rápido en aquellos pacientes que han consumido tabaco.

\section{CONCLUSIÓN}

El artículo ha trabajado una primera muestra de ochenta y tres pacientes que han acudido a la clínica "Haro Dental" en Haro (La Rioja-España) y que han permitido que sus datos sean tratados con una finalidad académica. A pesar de las limitaciones que tiene trabajar con una muestra tan pequeña y dividirla en submuestras más pequeñas, la investigación ha conseguido unos resultados relevantes y llamativos.

El primer resultado que cabe destacar es que la literatura sobre la incidencia del tabaco se ha centrado en un grupo de enfermedades y afecciones dejando en la marginalidad otra clase de investigaciones como los efectos del tabaco en la salud oral de los pacientes. La localización de estas investigaciones, así como su repercusión es muy pequeña, cuando los efectos son más visibles en el consumidor de tabaco.

El segundo resultado se obtiene gracias a la base de datos de la Dra. Ibañez, responsable de la clínica "Haro Dental". Con esta pequeña muestra se ve con claridad que los pacientes que consumen tabaco tienen una peor salud oral, si hablamos de caries. Esta diferencia no se ve si se estudian otras variables como sexo, edad o localidad.

Las limitaciones de la base de datos son ob- vias. La primera el número de individuos de la muestra. Al generar submuestras de menos de 51 individuos no ha permitido realizar estudios de significatividad, ya que los instrumentos no hubieran servido con una muestra tan pequeña (TorresRemírez, 2018). Sin embargo, la hipótesis lanzada y defendida en este estudio, hasta donde conocen los autores, no ha sido estudiada para los adultos. Únicamente se ha encontrado un estudio que relaciona el tabaco de mascar con las caries en adultos de Estados Unidos (Tommar y Winn, 1999). Por lo que convierte este artículo en el primero en tratar la relación de todas las clases de tabaco con las caries, tanto a nivel nacional como internacional.

Los retos de futuras investigaciones serán conseguir de una misma área geográfica delimitada un mayor número de individuos y una desagregación mayor, tanto por clases de tabaco consumido como por nivel de educación. Con ello se podrá hacer un estudio más completo de los efectos del tabaco en la salud oral.

TORRES-REMÍREZ, J. \& IBÁÑEZ, M. The relationship between oral health and tobacco consumption. A study of the inhabitants of La Rioja Alta. Int. J. Odontostomat., 14(1):95-100, 2020.

ABSTRACT: Studying the effects of tobacco use (addictive product) on the health of patients continues to be a priority for society. After reading " La Historia Medicinal de las cosas que se traen de nuestras Indias Occidentales " by Nicolás Monardes, a list of all the benefits attributed to tobacco was made. One of the few that has not been refuted is the use of tobacco for toothache. Therefore, the objective of the study is to relate the consumption of snuff with oral health (caries) to replicate the claim of Monardes. A pilot study with patients who visit the dentist in La Rioja Alta (Spain) was carried out. Permission was sought from the individuals who came to discuss their personal data in the study. Descriptive statistics was used. Eighty-three patients gave their data for the study, with the majority being women $(59.13 \%)$. The majority are not tobacco consumers; tobacco users are in their entirety daily smokers. The average number of caries of the population is 2.74 , being that of smokers 3.90 and that of non-smokers 2.10. The low number of the sample does not allow a study of significance. However, initial results show that smokers have worse oral health, which leads them to have more cavities. This difference is not critical if the study includes other variables (sex, location or age).

KEY WORDS: tobacco, caries, Spain. 


\section{REFERENCIAS BIBLIOGRÁFICAS}

AECC. Tabaquismo y cáncer en España. Situación Actual Observatorio del Cáncer de la Asociación Española Contra el Cáncer (AECC), 2018

Barrado, B. \& Torres-Remírez, J. Oncobarómetro. Percepción de la gravedad de las enfermedades, de sus posibilidades de curación y de las causas que provocan su temor en función de las características socioeconómicas. Observatorio del Cáncer de la Asociación Española Contra el Cáncer (AECC), 2015.

Bertazzo-Silveira, E.; Maikel Kruger, C.; Porto De Toledo, I.; Porporatti, A.; Dick, B.; Flores-Mir, C. \& De Luca Canto, G. Association between sleep bruxism and alcohol, caffeine, tobacco and drug abuse. J. Am. Dental Assoc., 147(11):859-66, 2016.

Cuba Díaz, Y.; García Saborit, V.; Rodríguez Guerra, Y.; Gómez Mariño, M. \& Saborit Casanovas, V. El tabaquismo como factor de riesgo de enfermedades bucales" Rev. Fund. Juan José Carraro, 32:20-9, 2010.

Gayoso Aguirre, J. C. Efecto del tabaquismo sobre la tasa del flujo salival en pacientes de 25 a 60 años que acuden al hospital materno infantil Carlos Showing Ferrari Huánuco 2017. Tesis para Optar el título Profesional de Cirujano Dentista. Huanuco, Universidad de Huánuco, 2017.

Míguez Varela, M. C. \& Becoña, E. ¿El consumo de cigarrillos y alcohol se relaciona con el consumo de cánnabis y el juego problema en adolescentes españoles? Adicciones, 27(1):8-16, 2015.

Monardes, N. La historia Medicinal de las cosas que se traen de nuestras Indias Occidentales (1565-1574). Madrid, Ministerio de Sanidad y Consumo, España, 1992.

Tommar, S. L. \& Winn, D. M. Chewing tobacco use and dental caries among US men. J. Am. Dental Assoc., 130(11):1601-10, 1999.

Torres-Remírez, J. \& Remírez, M. T. Monardes o el olvido de un humanista. Rev. Investig. Hum., UFM-RIHU, 5:1-19, 2018.

Torres-Remírez, J.; Salguero-Chaves, E.; Torres-Remírez, M. \& Regla, R. ¿Es el nivel de ingreso del paciente un factor relevante a la hora de dejar de fumar? Rev. Col. Méd. Cir. Guatemala (COLMEDEGUA), 157(2):66-9, 2018.

Villalbí, J. El tabaco como problema de Salud Pública. Inf. SESPAS, 7:113-29, 2002
Direccion para correspondencia a: José Torres-Remírez Universidad de Zaragoza ESPAÑA

\section{Email: josetorres@unizar.es}

Recibido : 07-08-2019

Aceptado: 13-09-2019 